PROCEEDINGS OF THE

AMERICAN MATHEMATICAL SOCIETY

Volume 137, Number 1, January 2009, Pages 273-278

S 0002-9939(08)09572-5

Article electronically published on August 4, 2008

\title{
A NOTE ON RICCI SIGNATURES
}

\author{
DEZHONG CHEN
}

(Communicated by Jon G. Wolfson)

\begin{abstract}
We show that only two types of Ricci signatures cannot be realized by any left-invariant metric on 4-dimensional Lie groups.
\end{abstract}

\section{INTRODUCTION}

On an $n$-dimensional manifold, $n \geq 3$, one can ask whether there is a complete Riemannian metric whose Ricci curvature has a given signature, i.e. a given number of positive, zero and negative eigenvalues. It is well-known that such a prescribed Ricci signature problem does not always have a solution. For example, the classical Bonnet-Myers theorem says that for a complete Riemannian manifold to have positive Ricci curvature, it must be compact and its fundamental group has to be finite. Another standard example is the Cheeger-Gromoll splitting theorem, which gives topological obstructions to the existence of a complete metric of nonnegative Ricci curvature. On the other hand, J. Lohkamp ([5]) showed that any manifold of dimension $\geq 3$ admits a complete metric of negative Ricci curvature. However, there seems to be little knowledge about Ricci signatures with mixed signs. Here, we just want to mention D. M. DeTurck's paper [1, which implies the local solvability of the prescribed Ricci signature problem in the absence of zeroes.

In this note, we restrict our attention to Lie groups with left-invariant metrics and study their Ricci signatures. This kind of problem has been attacked by many authors before. In his beautiful survey article [7, J. Milnor classified Ricci signatures of left-invariant metrics on 3-dimensional Lie groups and found that there are three types of Ricci signatures which cannot be realized by any left-invariant metric on 3-dimensional Lie groups. Based on his work, Milnor raised the problem of seeking possible restrictions on Ricci signatures of left-invariant metrics in higher dimensions. In 2, I. Dotti-Miatello determined Ricci signatures of left-invariant metrics on two-step solvable unimodular Lie groups.

Motivated by the works mentioned above, we consider the realization of Ricci signatures of left-invariant metrics on 4-dimensional Lie groups. First of all, we note that there are, in total, fifteen candidates for Ricci signatures of left-invariant metrics on 4-dimensional Lie groups, as indicated in Table 1 .

Remark 1.1. It follows from the results of Milnor ([7], §4) that Ricci signatures $S_{1}$ to $S_{7}$ can be realized by left-invariant product metrics on product Lie groups of

Received by the editors December 18, 2007.

2000 Mathematics Subject Classification. Primary 53C21.

Key words and phrases. Left-invariant metric, Ricci signature.

(C)2008 American Mathematical Society Reverts to public domain 28 years from publication 
a 3-dimensional Lie group with $S^{1}$. For example, according to Milnor ([7, Corollary 4.5), Ricci signatures $S_{1}, S_{4}$ and $S_{6}$ can be realized by left-invariant product metrics on $S U(2) \times S^{1}$. However, this construction fails to give examples of leftinvariant metrics with Ricci signatures $S_{12}, S_{14}$ or $S_{15}$, as there are no 3-dimensional Lie groups admitting left-invariant metrics with Ricci signatures $(+,+, 0),(+,+,-)$ or $(+, 0,-)$.

TABLE 1. List of Ricci signatures of left-invariant metrics

\begin{tabular}{|l|c|l|c|l|c|}
\hline Name & Description & Name & Description & Name & Description \\
\hline$S_{1}$ & $(+,+,+, 0)$ & $S_{2}$ & $(0,0,0,0)$ & $S_{3}$ & $(0,-,-,-)$ \\
\hline$S_{4}$ & $(+, 0,0,0)$ & $S_{5}$ & $(0,0,0,-)$ & $S_{6}$ & $(+, 0,-,-)$ \\
\hline$S_{7}$ & $(0,0,-,-)$ & $S_{8}$ & $(+,+,-,-)$ & $S_{9}$ & $(+,-,-,-)$ \\
\hline$S_{10}$ & $(-,-,-,-)$ & $S_{11}$ & $(+,+,+,+)$ & $S_{12}$ & $(+,+, 0,0)$ \\
\hline$S_{13}$ & $(+,+,+,-)$ & $S_{14}$ & $(+,+, 0,-)$ & $S_{15}$ & $(+, 0,0,-)$ \\
\hline
\end{tabular}

Remark 1.2. It follows from the results of Dotti-Miatello ([2, Proposition 4.1) that Ricci signatures $S_{2}$ and $S_{5}$ to $S_{9}$ can be realized by left-invariant metrics on 4dimensional two-step solvable unimodular Lie groups. However, his results do not afford examples of left-invariant metrics with Ricci signatures $S_{10}$ to $S_{15}$.

Remark 1.3. It follows from Jensen's classification of 4-dimensional Lie groups with left-invariant Einstein metrics ([4, p. 348) that Ricci signature $S_{10}$ can be realized by left-invariant Einstein metrics on 4-dimensional solvable Lie groups.

From the above three remarks, we know that Ricci signatures $S_{1}$ to $S_{10}$ can be realized by left-invariant metrics on 4 -dimensional Lie groups. Now we are left with Ricci signatures $S_{11}$ to $S_{15}$. An easy observation asserts that Ricci signature $S_{11}$ cannot be realized at all; otherwise the underlying Lie group $G$ would have positive Ricci curvature. Hence its Lie algebra $\mathfrak{g}$ must be equal to its commutator ideal $[\mathfrak{g}, \mathfrak{g}]$ ([7], Lemma 2.3); i.e. $\mathfrak{g}$ would be perfect. This contradicts the fact that there is no 4-dimensional perfect Lie algebra ([8], Table 1, p. 988). Besides this observation, we have the following

Theorem 1.4. No 4-dimensional Lie group admits a left-invariant metric with Ricci signature $S_{12}$.

The complete proof of Theorem 1.4 will be given in $\S 2$. However, it is instructive to see why Theorem 1.4 is true in some special cases. Let $(G, g)$ be a 4 -dimensional Lie group with a left-invariant metric of Ricci signature $S_{12}$. Then $(G, g)$ has nonnegative Ricci curvature. Hence we can apply to $(G, g)$ the Cheeger-Gromoll splitting theorem and assume that $(G, g)$ is a Riemannian product $\left(\bar{G} \times \mathbb{R}^{k}, \bar{g} \times g_{0}\right)$, where $g_{0}$ is the canonical flat metric of $\mathbb{R}^{k}$ and $(\bar{G}, \bar{g})$ is a $(4-k)$-dimensional Lie group with a left-invariant metric of nonnegative Ricci curvature. Note that $0 \leq k \leq 2$ as the Ricci curvature of $(G, g)$ has exactly two zero eigenvalues. If $k=2$, then $\left(\bar{G}^{2}, \bar{g}\right)$ has positive Ricci curvature; i.e. $(\bar{G}, \bar{g})$ has positive scalar curvature, which is impossible since $\bar{G}$ is solvable ([7], Theorem 3.1). If $k=1$, then $\left(\bar{G}^{3}, \bar{g}\right)$ has Ricci signature $(+,+, 0)$. This also gives a contradiction. (See Remark [1.1)

In $\S 3$, we classify Ricci signatures of left-invariant metrics on $S U(2) \times S^{1}$. In particular, we show that there exist left-invariant metrics of Ricci signatures $S_{13}$ to $S_{15}$. 
We hope that our results can be applied to Ricci flow on 4-dimensional Lie groups. For this direction, see [3] and references therein.

The author wishes to express his gratitude to his Ph.D. supervisor, Professor McKenzie Wang, for his constant encouragement and valuable discussions of mathematics in general and of differential geometry in particular. The author is also grateful to Xuping Zhang for her support and help in preparing the manuscript.

\section{Proof of Theorem 1.4}

Let $(G, g)$ be a 4 -dimensional Lie group with a left-invariant metric, and let $\mathfrak{g}$ be the associated Lie algebra, consisting of all smooth left-invariant vector fields on $G$. Assume that $(G, g)$ has Ricci signature $S_{12}$. By definition, $(G, g)$ has nonnegative Ricci curvature. Thus $G$ is unimodular (77, Lemma 6.4). Moreover, $G$ cannot be solvable; otherwise $g$ would be flat ([7, Theorem 3.1) and hence Ricci flat, which contradicts our Ricci signature assumption. Now we look at the classification of 4-dimensional unimodular Lie algebras ([6], pp 306-307). It turns out that only two of these are not solvable; their brackets are given as follows, where $\left\{X_{i}\right\}_{1}^{4}$ is a basis of the Lie algebra. Here we adopt the notation in 6 .

(1) Class U3S1

$$
\begin{array}{lll}
{\left[X_{1}, X_{4}\right]=0} & {\left[X_{2}, X_{4}\right]=0} & {\left[X_{3}, X_{4}\right]=0} \\
{\left[X_{2}, X_{3}\right]=X_{1}} & {\left[X_{3}, X_{1}\right]=X_{2}} & {\left[X_{1}, X_{2}\right]=-X_{3}}
\end{array}
$$

This Lie algebra is isomorphic to $\mathfrak{s l}(2, \mathbb{R}) \oplus \mathbb{R}$, whose derived algebra is isomorphic to $\mathfrak{s l}(2, \mathbb{R})$.

(2) Class U3S3

$$
\begin{array}{lll}
{\left[X_{1}, X_{4}\right]=0} & {\left[X_{2}, X_{4}\right]=0} & {\left[X_{3}, X_{4}\right]=0} \\
{\left[X_{2}, X_{3}\right]=X_{1}} & {\left[X_{3}, X_{1}\right]=X_{2}} & {\left[X_{1}, X_{2}\right]=X_{3}}
\end{array}
$$

This Lie algebra is isomorphic to $\mathfrak{s u}(2) \oplus \mathbb{R}$, whose derived algebra is isomorphic to $\mathfrak{s u}(2)$.

In both cases, the Lie algebra $\mathfrak{g}$ is the direct sum $\mathfrak{g}^{\prime} \oplus \mathbb{R} X_{4}$, where $\mathfrak{g}^{\prime}$, spanned by $\left\{X_{i}\right\}_{1}^{3}$, is the derived algebra of $\mathfrak{g}$ and $X_{4}$ is a center element. Moreover, $\mathfrak{g}^{\prime}$ is unimodular. So there exists an orthonormal basis $\left\{e_{i}\right\}_{1}^{3}$ of $\mathfrak{g}^{\prime}$ such that

$$
\left[e_{2}, e_{3}\right]=\lambda_{1} e_{1}, \quad\left[e_{3}, e_{1}\right]=\lambda_{2} e_{2}, \quad\left[e_{1}, e_{2}\right]=\lambda_{3} e_{3} .
$$

(7], pp 305-307). As pointed out by Milnor, by changing signs if necessary, we may assume that at most one of the structure constants $\left\{\lambda_{i}\right\}$ is negative. Therefore, for $\mathfrak{s l}(2, \mathbb{R})$ and $\mathfrak{s u}(2)$, the signs of $\left\{\lambda_{i}\right\}$ are of types $(+,+,-)$ and $(+,+,+)$, respectively. In particular, $\lambda_{1} \lambda_{2} \lambda_{3} \neq 0$. Now we choose $e_{4}$ to be a unit left-invariant vector field perpendicular to $\mathfrak{g}^{\prime}$. Thus $\left\{e_{i}\right\}_{1}^{4}$ becomes an orthonormal basis of $\mathfrak{g}$. Therefore we can write $X_{4}$ as a linear combination of $\left\{e_{i}\right\}_{1}^{4}$, say

$$
X_{4}=a e_{1}+b e_{2}+c e_{3}+d e_{4} .
$$

Note that $d \neq 0$, otherwise $X_{4} \in \mathfrak{g}^{\prime}$, which gives a contradiction. For the sake of simplicity, we assume that $d=1$. Then we can express $e_{4}$ as a linear combination of $\left\{e_{1}, e_{2}, e_{3}, X_{4}\right\}$, i.e.

$$
e_{4}=X_{4}-a e_{1}-b e_{2}-c e_{3} .
$$

This allows us to compute Lie brackets $\left[e_{i}, e_{4}\right], i=1,2,3$. The results are as follows:

$$
\left[e_{1}, e_{4}\right]=c \lambda_{2} e_{2}-b \lambda_{3} e_{3}, \quad\left[e_{2}, e_{4}\right]=a \lambda_{3} e_{3}-c \lambda_{1} e_{1}, \quad\left[e_{3}, e_{4}\right]=b \lambda_{1} e_{1}-a \lambda_{2} e_{2} .
$$


With these brackets in hand, it is tedious but straightforward to compute the Ricci curvature $R_{i j}$. The results are as follows:

$$
\begin{aligned}
& R_{11}=\frac{1}{2}\left(\left(1+b^{2}+c^{2}\right) \lambda_{1}^{2}-\left(1+b^{2}\right) \lambda_{3}^{2}-\left(1+c^{2}\right) \lambda_{2}^{2}+2 \lambda_{2} \lambda_{3}\right) \\
& R_{22}=\frac{1}{2}\left(\left(1+a^{2}+c^{2}\right) \lambda_{2}^{2}-\left(1+c^{2}\right) \lambda_{1}^{2}-\left(1+a^{2}\right) \lambda_{3}^{2}+2 \lambda_{1} \lambda_{3}\right) \\
& R_{33}=\frac{1}{2}\left(\left(1+a^{2}+b^{2}\right) \lambda_{3}^{2}-\left(1+b^{2}\right) \lambda_{1}^{2}-\left(1+a^{2}\right) \lambda_{2}^{2}+2 \lambda_{1} \lambda_{2}\right) \\
& R_{44}=-\frac{1}{2}\left(a^{2}\left(\lambda_{2}-\lambda_{3}\right)^{2}+b^{2}\left(\lambda_{3}-\lambda_{1}\right)^{2}+c^{2}\left(\lambda_{1}-\lambda_{2}\right)^{2}\right) \\
& R_{12}=\frac{1}{2} a b\left(\lambda_{3}^{2}-\lambda_{1} \lambda_{2}\right) \quad R_{13}=\frac{1}{2} a c\left(\lambda_{2}^{2}-\lambda_{1} \lambda_{3}\right) \quad R_{23}=\frac{1}{2} b c\left(\lambda_{1}^{2}-\lambda_{2} \lambda_{3}\right) \\
& R_{14}=\frac{1}{2} a\left(\lambda_{3}-\lambda_{2}\right)^{2} \quad R_{24}=\frac{1}{2} b\left(\lambda_{3}-\lambda_{1}\right)^{2} \quad R_{34}=\frac{1}{2} c\left(\lambda_{1}-\lambda_{2}\right)^{2}
\end{aligned}
$$

Note that $R_{44} \leq 0$. Hence it follows from our Ricci signature assumption that $R_{44}=0$, i.e.

$$
a^{2}\left(\lambda_{2}-\lambda_{3}\right)^{2}+b^{2}\left(\lambda_{3}-\lambda_{1}\right)^{2}+c^{2}\left(\lambda_{1}-\lambda_{2}\right)^{2}=0 .
$$

There are three cases:

(1) $\lambda_{1}=\lambda_{2}=\lambda_{3}$

(2) $\lambda_{1}=\lambda_{2}, \lambda_{1} \neq \lambda_{3}$

(3) $\lambda_{1} \neq \lambda_{2}, \lambda_{1} \neq \lambda_{3}, \lambda_{2} \neq \lambda_{3}$

In case (1), $\left\{e_{i}\right\}$ diagonalizes the Ricci curvature, and we have

$$
R_{11}=R_{22}=R_{33}=\frac{1}{2} \lambda_{1}^{2}>0, \quad R_{44}=0 .
$$

In particular, the Ricci signature is $S_{1}$.

In case (2), it follows from (2.1) that $a=b=0$; hence $\left\{e_{i}\right\}$ also diagonalizes the Ricci curvature. Moreover, we have

$$
R_{11}=R_{22}=\lambda_{1} \lambda_{3}-\frac{1}{2} \lambda_{3}^{2}, \quad R_{33}=\frac{1}{2} \lambda_{3}^{2}>0, \quad R_{44}=0 .
$$

Depending on the choice of $\lambda_{i}$ 's, the Ricci signature can be either $S_{1}$ or $S_{4}$ or $S_{6}$. However, none is of type $S_{12}$.

In case (3), it follows from (2.1) that $a=b=c=0$; hence $\left\{e_{i}\right\}$ diagonalizes the Ricci curvature too. Moreover, we have

$$
\begin{array}{ll}
R_{11}=\frac{1}{2}\left(\lambda_{1}+\lambda_{2}-\lambda_{3}\right)\left(\lambda_{1}-\lambda_{2}+\lambda_{3}\right) & R_{22}=\frac{1}{2}\left(\lambda_{1}+\lambda_{2}-\lambda_{3}\right)\left(\lambda_{2}+\lambda_{3}-\lambda_{1}\right) \\
R_{33}=\frac{1}{2}\left(\lambda_{1}-\lambda_{2}+\lambda_{3}\right)\left(\lambda_{2}+\lambda_{3}-\lambda_{1}\right) & R_{44}=0
\end{array}
$$

Depending on the choice of $\lambda_{i}$ 's, the Ricci signature can be either $S_{1}$ or $S_{4}$ or $S_{5}$ or $S_{6}$. Again, none is of type $S_{12}$.

Therefore, we can conclude that there is no left-invariant metric of Ricci signature $S_{12}$ on Lie algebras of Classes U3S1 and U3S3. This completes the proof of Theorem 1.4 .

\section{RiCCi Signatures of LEFT-INVARIANT METRICS ON $S U(2) \times S^{1}$}

In this section, we exhibit numerical examples of left-invariant metrics of different Ricci signatures on $S U(2) \times S^{1}$ and show that these examples realize all possible Ricci signatures of left-invariant metrics on $S U(2) \times S^{1}$. Of particular interest is 
that among them there are left-invariant metrics of Ricci signatures $S_{13}$ to $S_{15}$. For this purpose, we introduce

Definition 3.1. We denote by $\mathfrak{g}^{4}\left(a, b, c, \lambda_{1}, \lambda_{2}, \lambda_{3}\right)$ the 4-dimensional metric Lie algebra admitting an orthonormal basis $\left\{e_{i}\right\}$ with multiplication table

$$
\begin{aligned}
& {\left[e_{1}, e_{2}\right]=\lambda_{3} e_{3} \quad\left[e_{2}, e_{3}\right]=\lambda_{1} e_{1} \quad\left[e_{3}, e_{1}\right]=\lambda_{2} e_{2}} \\
& {\left[e_{1}, e_{4}\right]=c \lambda_{2} e_{2}-b \lambda_{3} e_{3} \quad\left[e_{2}, e_{4}\right]=a \lambda_{3} e_{3}-c \lambda_{1} e_{1} \quad\left[e_{3}, e_{4}\right]=b \lambda_{1} e_{1}-a \lambda_{2} e_{2}}
\end{aligned}
$$

where $a, b, c, \lambda_{1}, \lambda_{2}, \lambda_{3}$ are parameters.

Note that the formulas for the Ricci curvature of $\mathfrak{g}^{4}\left(a, b, c, \lambda_{1}, \lambda_{2}, \lambda_{3}\right)$ have been given in $\S 2$.

Theorem 3.2. Depending on the choice of left-invariant metrics, the Ricci signature for $S U(2) \times S^{1}$ can be either $S_{1}$ or $S_{4}$ or $S_{6}$ or $S_{8}$ or $S_{9}$ or $S_{13}$ or $S_{14}$ or $S_{15}$.

Proof. According to Theorem 1.4 and the preceding argument in the Introduction, we may a priori rule out Ricci signatures $S_{11}$ and $S_{12}$ on $S U(2) \times S^{1}$. Since there is no left-invariant metric of nonpositive Ricci curvature on compact nonabelian Lie groups, we may also rule out Ricci signatures $S_{2}, S_{3}, S_{5}, S_{7}$ and $S_{10}$ on $S U(2) \times S^{1}$. On the other hand, we have already shown in Remark 1.1 that Ricci signatures $S_{1}$, $S_{4}$ and $S_{6}$ can be realized on $S U(2) \times S^{1}$. Now it remains to check the following statements:

(1) $\mathfrak{g}^{4}\left(0,1,1,1,1, \frac{7}{5}\right)$ has Ricci signature $S_{8}$.

(2) $\mathfrak{g}^{4}\left(0, \sqrt{\frac{3}{5}}, 2 \sqrt{5}, 1,1, \frac{8}{5}\right)$ has Ricci signature $S_{9}$.

(3) $\mathfrak{g}^{4}\left(0,1,1,1,1, \frac{4}{3}\right)$ has Ricci signature $S_{13}$.

(4) $\mathfrak{g}^{4}\left(0,1,1,1,1, \frac{1+\sqrt{3}}{2}\right)$ has Ricci signature $S_{14}$.

(5) $\mathfrak{g}^{4}\left(0, \sqrt{\frac{3}{5}}, 2 \sqrt{5}, 1,1, \frac{3}{2}\right)$ has Ricci signature $S_{15}$.

In the above five explicit examples, the signs of the constants $\left\{\lambda_{i}\right\}$ are $(+,+,+)$. Hence it follows from the proof of Theorem 1.4 that we can choose $S U(2) \times S^{1}$ as the underlying Lie group. This completes the proof.

\section{REFERENCES}

1. D. M. DeTurck, Existence of metrics with prescribed Ricci curvature: local theory, Invent. Math. 65 (1981/82), no. 1, 179-207. MR636886(83b:53019)

2. I. Dotti-Miatello, Ricci curvature of left invariant metrics on solvable unimodular Lie groups, Math. Z. 180 (1982), no. 2, 257-263. MR661702 (84a:53044)

3. J. Isenberg, M. Jackson, P. Lu, Ricci flow on locally homogeneous closed 4-manifolds, Comm. Anal. Geom. 14 (2006), no. 2, 345-386. MR2255014 (2007e:53087)

4. G. R. Jensen, Homogeneous Einstein spaces of dimension four, J. Differential Geometry 3 (1969), 309-349. MR0261487 (41:6100)

5. J. Lohkamp, Metrics of negative Ricci curvature, Ann. of Math. (2) 140 (1994), no. 3, 655-683. MR.1307899 (95i:53042)

6. M. A. H. MacCallum, On the classification of the real four-dimensional Lie algebras, On Einstein's path (A. Harvey, ed.), Springer, New York, 1999, pp. 299-317. MR1658911|(99k:17010) 
7. J. Milnor, Curvatures of left invariant metrics on Lie groups, Advances in Math. 21 (1976), no. 3, 293-329. MR0425012 (54:12970)

8. J. Patera, R. T. Sharp, P. Winternitz, H. Zassenhaus, Invariants of real low dimension Lie algebras, J. Mathematical Phys. 17 (1976), no. 6, 986-994. MR0404362 (53:8164)

Department of Mathematics \& Statistics, Mcmaster University, Hamilton, Ontario L8S 4K1, CANADA

E-mail address: chend6@math.mcmaster.ca 